\title{
Indução de Diabetes Tipo 2 por dieta hiperlipídica e baixa dose de estrep- tozotocina em ratas wistar
}

\author{
Induction of Type 2 Diabetes by low dose of streptozotocin and \\ high-fat diet-fed in wistar rats
}

André M. Correia-Santos ${ }^{1}$, Akemi Suzuki², Juliana S. Anjos ${ }^{3}$, Thaís S. Rêgo ${ }^{4}$, Kátia C. L. Almeida ${ }^{5}$, Gilson T. Boaventura ${ }^{6}$

\begin{abstract}
RESUMO
O objetivo do presente estudo experimental foi desenvolver um modelo animal de diabetes tipo 2 que mimetizasse o curso natural e metabólico desta doença em humanos. Assim, foi oferecida uma dieta hiperlipídica (com aproximadamente $60 \%$ das calorias totais provenientes de lipídeos) por três semanas, estabelecendo então, um quadro de resistência à insulina. Em seguida, as ratas foram submetidas a uma dose única de estreptozotocina (STZ) $(35 \mathrm{mg} / \mathrm{kg}$ de peso corporal) em veículo de tampão citrato $(\mathrm{pH}: 4,4)$. Após 1 semana da injeção de STZ, as ratas foram submetidas ao teste oral de tolerância à glicose, mediante administração oral de glicose ( $2 \mathrm{~g}$ glicose/kg de massa corporal). Os dados foram submetidos à comparação entre os grupos utilizando-se o teste $t$. A significância em todos os testes se deu ao nível de $p \leq 0,05$. O grupo controle manteve a concentração média de glicose final igual a inicial. Após ingestão da dieta hiperlipídica houve um aumento da glicemia de jejum em cerca de $16,5 \%$ em relação ao momento inicial do experimento $(P<0,0001)$ enquanto os animais que receberam a dieta controle apresentaram um aumento aproximado de 8,7\%. Uma semana após a administração intraperitoneal de STZ, pode-se verificar aumento da concentração média de glicose no grupo hiperlipídico em cerca de $194,2 \%$, representando um aumento de $275,8 \%(P<0,0001)$ quando comparado ao GC. $O$ presente estudo retrata que a combinação de uma dieta hiperlipídica e baixa dose de estreptozotocina serve como um modelo animal alternativo para a diabetes tipo 2 simulando a síndrome em humanos.
\end{abstract}

Palavras-chave: Diabetes Mellitus Experimental. Estreptozotocina. Ratos Wistar. Dieta Hiperlipídica.

1. Pós-graduando (Doutorado) em Patologia da Faculdade de Medicina da Universidade Federal Fluminense (FM-UFF), Niterói, Rio de Janeiro.

2 Graduanda em Nutrição na UFF, Bolsista de Iniciação Científica do CNPQ.

3. Pós-graduanda (Mestrado) em Patologia da FM-UFF.

4. Pós-graduanda (Mestrado) em Ciência Médicas da FM-UFF.

5. Docente de Patologia, Faculdade de Medicina, Universidade Federal do Rio de Janeiro Campus UFRJ - Macaé.

6. Docente do Departamento de Nutrição e Dietética da Faculdade de Nutrição da UFF. Coordenador do Laboratório de Nutrição Experimental.
Correspondência: Laboratório de Nutrição Experimental. Rua Mario Santos Braga, 30, 5Andar, Valonguinho, CEP: 24020-140 - Niterói E-mail: andremcorreia@gmail.com

Artigo recebido em 14/05/2012 Aprovado para publicação em 27/08/2012 


\section{Introdução}

O Diabetes Tipo 2 é uma síndrome de etiologia múltipla, decorrente da falta de insulina e/ou da incapacidade da mesma de exercer adequadamente seus efeitos, resultando em resistência a este hormônio, seguida pela incapacidade das células $\beta$ em compensar tal resistência (disfunção das células $\beta$ pancreáticas). ${ }^{1,2}$ Esta síndrome caracteriza-se pela presença de hiperglicemia crônica, freqüentemente, acompanhada de dislipidemia, hipertensão arterial e disfunção endotelial. ${ }^{1}$

Por definição, a resistência à insulina é um estado de reduzida resposta a níveis normais deste hormônio. Geralmente este quadro desenvolve-se como primeiro indicador do diabetes tipo $2 .{ }^{3} \mathrm{O}$ pâncreas pode compensar a resposta reduzida à insulina, com o aumento de sua secreção, na tentativa de controlar a concentração de glicose no sangue. A hipersecreção contínua pode levar à falência das células $\beta$ do pâncreas resultando na menor produção deste hormônio e a manifestação do diabetes tipo $2 .^{3,4,5}$

Apesar do papel da predisposição genética, outros fatores como o envelhecimento, a obesidade, a qualidade da dieta e o estilo de vida sedentário são os principais fatores de risco envolvidos no desenvolvimento no diabetes tipo $2 .^{6}$

Os modelos animais de diabetes têm sido usados extensivamente na obtenção do esclarecimento sobre esta doença. Eles têm fornecido inúmeros benefícios para a saúde, incluído o tratamento de complicações oculares ou vasculares e o desenvolvimento de fármacos e de técnicas de transplante de pâncreas, entre outras. ${ }^{7}$ Embora exista um excedente de modelos animais (tanto espontâneos como induzidos) disponíveis para o estudo do diabetes tipo 2, o padrão de início da doença e o desenvolvimento da maioria deles não parece ser análogo à situação clínica em seres humanos. No entanto, existem certos modelos genéticos chamados ratos ZDF (Zucker Diabetic Fatty rats) e camundongos $\mathrm{db} / \mathrm{db}$ que desenvolvem diabetes espontaneamente, semelhantes a diabetes tipo 2 . O desenvolvimento do diabetes nesses animais é predominantemente determinado geneticamente, ao contrário do observado em seres humanos. Além disso, as observações derivadas destas linhagens geneticamente puras, não podem de forma satisfatória, ser extrapoladas para a população humana, devido à grande heterogeneidade dos humanos. Além disso, em modelos de indução de diabetes, a maioria dos animais (adultos ou recém-nascidos) requer doses relativamente altas de estreptozotocina (STZ >50mg.kg ${ }^{-1}$ ) (glicosamina-nitrosureia, antibiótico com propriedades diabetogênicas). O desenvolvimento da hiperglicemia nesses ratos após a injeção de STZ é conseqüente da destruição direta das células $\beta$ pancreáticas e resulta na deficiência de insulina. Desta forma, a hiperglicemia observada no caso não é decorrente de um quadro de resistência insulínica. Assim, esses modelos mostram mais sintomas e características típicas de diabetes tipo 1 do que diabetes do tipo $2 \mathrm{e}$; ainda não são muito sensíveis aos efeitos de drogas, como os insulinotrópicos e sensibilizadores de insulina. Em contraste, ratos alimentados com dieta rica em gordura (dieta hiperlipídica) desenvolvem obesidade, hiperinsulinemia e resistência à insulina, mas não desenvolvem hiperglicemia alta, limitando assim, a triagem de agentes que controlam as concentrações de glicose no sangue. ${ }^{2,8}$

Visto o exposto, este estudo teve como objetivo desenvolver um modelo metabólico de diabetes tipo 2 em ratos wistar que mimetiza o curso natural e metabólico da doença em humanos (de resistência à insulina à disfunção da célula $\beta$ ).

\section{Material e métodos}

\section{Delineamento Experimental}

Todos os procedimentos experimentais realizados seguiram as normas estabelecidas no guia convencional para a experimentação animal (Publicação NIH No.85-23, revisado em 1996) e as recomendações nacionais impostas pela Lei Arouca (11.794/2008). O projeto foi submetido e aprovado pelo Comitê de Ética em Experimentação Animal do Núcleo de Animais de Laboratório da Universidade Federal Fluminense com o número de registro: 035/2011.

Foram utilizadas 30 ratas wistar provenientes da colônia do Laboratório de Nutrição Experimental (LabNE) da UFF, nulíparas, com aproximadamente três meses de idade e foram mantidas em condições controladas de temperatura $\left(22 \pm 2^{\circ} \mathrm{C}\right)$ e umidade $(60$ $\pm 10 \%$ ), com acesso irrestrito a comida e água, e com ambiente submetido a ciclos de 12:12h claro-escuro.

Do total de animais utilizados, 24 ratas foram alimentadas com dieta de alta densidade energética (hiperlipídica), pelo período inicial de três semanas, e seis ratas foram alimentadas com dieta à base de caseína, ambas ad libitum, formando então dois grupos: Grupo Hiperlipídico $(\mathrm{n}=24)$ e Grupo Controle $(\mathrm{n}=6)$. A composição da dieta hiperlipídica e da dieta controle está descrita na Tabela 1. 


\section{Tabela 1}

Composição a cada 1000g das dietas utilizadas durante o ensaio

\begin{tabular}{|c|c|c|}
\hline Ingrediente/ Dieta & Dieta controle & Dieta hiperlipídica \\
\hline Caseína $(\mathrm{g}) *$ & 140 & 190 \\
\hline Amido de milho $(\mathrm{g}) \dagger$ & 619,492 & 249,492 \\
\hline Sacarose $(g) \neq$ & 100 & 100 \\
\hline Óleo de soja $(\mathrm{ml}) * *$ & 40 & 40 \\
\hline Banha de porco (g) $¥$ & - & 320 \\
\hline Fibra $(g) \dagger \dagger$ & 50 & 50 \\
\hline Mix de vitaminas (AIN93-M) $(\mathrm{g})^{\S}$ & 10 & 10 \\
\hline Mix de minerais (AIN93-M) $(\mathrm{g}) \S$ & 35 & 35 \\
\hline Cistína $(\mathrm{g}) \phi$ & 3 & 3 \\
\hline Colina $(g) \phi$ & 2,5 & 2,5 \\
\hline BHT (Terc-butilhidroquinona) (g) & 0,008 & 0,008 \\
\hline Total $(\mathrm{g})$ & 1000 & 1000 \\
\hline Carboidrato $(\%)$ & 76 & 26 \\
\hline Proteína (\%) & 14 & 14 \\
\hline Lipídio (\%) & 10 & 60 \\
\hline Energia (Kcal/kg) & 3573 & 5404 \\
\hline $\begin{array}{l}\text { AIN-93M = American Institute of Nu } \\
* \text { Comércio e Indústria Farmos Ltda } \\
\text { † Maizena da Unilever Bestfoods Br } \\
\text { † União (Rio de Janeiro, RJ, Brasil). }\end{array}$ & $\begin{array}{l}\text { iro RJ, Brasil). } \\
\text { logi Guaçu, SP, Br }\end{array}$ & \\
\hline \multicolumn{3}{|c|}{ ** Liza da Cargill Agricultura Ltda. (Mairinque, SP, Brasil). } \\
\hline \multicolumn{3}{|c|}{$\dagger \dagger$ Microcel da Blanver Ltda. (Cotia, SP, Brasil). } \\
\hline \multicolumn{3}{|c|}{$¥$ Sadia Comercial Ltda } \\
\hline \multicolumn{3}{|c|}{ § PragSoluções Comércio e Serviços Ltda-ME (Jáu, SP, São Paulo) } \\
\hline$\phi$ M. Cassab Comércio e Indústria Ltd & SP, Brasil) & \\
\hline
\end{tabular}

Depois de três semanas da dieta hiperlipídica, as ratas receberam injeção intraperitoneal (i.p.) de estreptozotocina (STZ) em baixa dosagem (35 mg. $\left.\mathrm{kg}^{-1}\right)$ dissolvida em veículo (solução de citrato de sódio $0,01 \mathrm{M}, \mathrm{pH}=4,5$ ) segundo Sriniva-san et al. ${ }^{2}$ As ratas que consumiram dieta controle receberam somente o veículo por via intraperitoneal.

Estudos prévios, empregando desenho experimental semelhante ao nosso, realizados por Srinivasan et al. ${ }^{2}$ e Mu et al. ${ }^{8}$, utilizando ratos Sprague-Dawley, mostraram que a dieta hiperlipídica ou a estreptozotocina isolada não afetaram significativamente a glicemia, enquanto que a combinação da dieta hiperlipídica e estreptozotocina, na dose de $35 \mathrm{mg} / \mathrm{kg}$, levou à fran- ca hiperglicemia. Foi mostrado também por Mu et al. ${ }^{8}$ que somente a dose de STZ causou uma redução de aproximadamente $40 \%$ dos níveis de insulina no plasma, o que não foi suficiente para causar a hiperglicemia nos animais alimentos com a dieta normal. Decidimos, portanto, somente utilizarmos dois grupos, um com a associação da dieta hiperlipídica e estreptozotocina (grupo hiperlipídico) e um só com dieta controle (Grupo controle).

O peso corporal e a glicose plasmática foram analisados antes da administração da dieta hiperlipídica e ao final das três semanas de administração da dieta, antes da injeção da STZ e depois de 7 dias da injeção, isto é, com 4 semanas de administração da dieta nas 
ratas. A confirmação do diabetes foi feita seguindo os seguintes critérios: concentração de glicose plasmática máxima maior que $288 \mathrm{mg} / \mathrm{dL}$, ou concentração maior que $198 \mathrm{mg} / \mathrm{dL}$ após 120 minutos de administração de solução oral de glicose ( $2 \mathrm{~g}$ glicose $/ \mathrm{kg}$ de massa corporal). ${ }^{9}$

\section{Avaliações metabólicas}

O teste oral de tolerância à glicose foi realizado ao final do experimento. Após 6 horas de jejum, uma amostra de sangue foi retirada da veia caudal de cada rato (tempo 0), que na seqüência recebia, via gavagem, uma solução de glicose na concentração de dois gramas de glicose por quilograma de peso. A glicemia foi determinada nos tempos zero (antes da administração de glicose), 15, 30, 60, 90 e 120 minutos após a injeção, utilizando-se glicosímetro e fitas para glicemia da marca Accu-Chek Active (Roche Diagnostics, Alemanha). A área sob a curva (AUC) foi calculada usando o GraphPad Prism versão 5.03 para Windows.

\section{Avaliação do valor biológico das rações}

Para a avaliação do valor biológico das rações, foi utilizado índice de eficiência alimentar, a partir do qual, foi possível verificar o quanto a ingestão energética foi eficiente em promover o aumento do peso dos animais. ${ }^{10}$ A ingestão de ração (g/dia) foi multiplicada pela energia da ração $(\mathrm{kcal} / \mathrm{g})$ para se obter a ingestão de energia diária por rato. A eficiência alimentar foi calculada como [(ganho de massa corporal $/ \mathrm{kcal}$ ingerida) $\mathrm{x} 100]$.

\section{Análise estatística}

Os dados foram apresentados na forma de média \pm erro padrão. A distribuição normal dos valores encontrados foi analisada através do teste Kolmogorov-Smirnov. Verificando-se a normalidade dos dados, estes foram submetidos à comparação entre os grupos utilizando-se o teste $t$ de Student para dados independentes. Nos resultados que não seguiram a distribuição normal foi aplicado o teste não paramétrico de Mann-Whitney. A significância em todos os testes foi estabelecida ao nível de $\mathrm{p} \leq 0,05$. Tais análises estatísticas foram realizadas pelo programa GraphPadPrism versão 4.03 para Windows (GraphPad Software, São Diego, Califórnia, EUA).

\section{Resultados}

\section{Ingestão de ração, energia e eficiência ali- mentar}

Até a terceira semana, o grupo hiperlipídico ingeriu $15 \%$ a menos de ração do que o grupo controle, porém a ingestão energética foi maior $(+30 \%$, $P<0,0001$ ) (Tabela 2). A administração da STZ ao grupo hiperlipídico e veículo ao grupo controle, resultou na diminuição da ingestão de ração em ambos os grupos, e diferente do momento pré STZ, os grupos passaram a ingerir a mesma quantidade de ração; contudo a ingestão de energia do grupo hiperlipídico continuou sendo superior ao grupo controle $(+43 \%, P=$ 0,0003) (Tabela 2).

Tabela 2

Ingestão de ração e energia.

Ração (g/dia/animal) Energia (Kcal/dia/animal)

Pré STZ ou veículo

Grupo Controle ( $n=6)$

$19,2 \pm 0,6 \quad 68,4 \pm 2,0$

Grupo Hiperlipídico $(n=24)$

$16,3 \pm 0,3^{\mathrm{a}} \quad 88,3 \pm 1,8^{\mathrm{a}}$

Pós STZ ou veículo

Grupo Controle $(n=6)$

$15,2 \pm 0,9^{¥}$

$54,1 \pm 3,3^{¥}$

Grupo Hiperlipídico $(n=24)$

$14,3 \pm 0,6^{\ddagger}$

$77,2 \pm 3,1^{\mathrm{a}} ¥$

Os dados são apresentados na forma de média \pm EPM.

A letra (a) representa diferença estatística comparada do Grupo Controle, $P<0,0001$. O símbolo (¥) representa diferença estatística quando comparado ao seu respectivo grupo pré $\mathrm{STZ}$ ou veículo, $P<0,001$.

STZ = Estreptozotocina 
A eficiência alimentar dos animais alimentados tanto com dieta controle quanto com dieta hiperlipídica foi igual no período pré STZ ou veículo (GC: $44,5 \% \pm 7,9 \%$ e GH: $41,7 \% \pm$ 1,8\%) (Figura 1). O mesmo não aconteceu após a administração da STZ no grupo hiperlipídico, que diminuiu a eficiência energética, de forma a torná-la negativa (GC: $31,7 \% \pm 4,2 \%$ e GH: $11,99 \% \pm 4,5 \%)$.

\section{Massa corporal}

Os animais iniciaram o experimento sem diferenças na massa corporal $(223,5 \mathrm{~g} \pm 2,5 \mathrm{~g}$ aos 3 meses de idade) (Figura 2). Ao final das três semanas de administração das dietas experimentais, não houve diferença estatisticamente significativa para este parâmetro (GC: $255 \mathrm{~g} \pm 21,6 \mathrm{~g}$ e GH: $269 \pm 19,1 \mathrm{~g})$.

A administração da STZ impediu a evolução do ganho de massa corporal e induziu a perda de massa no grupo hiperlipídico, enquanto que a administração de veículo ao grupo controle não causou alteração no ganho de massa corporal (Figura 2).

\section{Metabolismo Glicídico}

A ingestão da dieta hiperlipídica levou ao aumento da glicemia de jejum em cerca de $16,5 \%$ quando comparada ao momento basal do experimento $(P<0,0001)$ (Inicial: $100,5 \pm 2,8 \mathrm{mg} / \mathrm{dL}$; Final: $117 \pm 2,6 \mathrm{mg} / \mathrm{dL}$ ); enquanto que o grupo controle teve um aumento de aproximadamente $8,7 \%$ (Inicial: 105,8 $\pm 6,7 \mathrm{mg} / \mathrm{dL}$; Final: $115 \pm 2,3 \mathrm{mg}$ / dL) (Figura 3). Uma semana após a injeção intraperitoneal de STZ, foi observado aumento da concentração média de glicose de aproximadamente $194,2 \%$ no grupo hiperlipídico (344,2 \pm $6,3 \mathrm{mg} / \mathrm{dL}$ ) e quando comparado ao grupo controle $(91,6 \pm 2,6 \mathrm{mg} / \mathrm{dL})$, a STZ levou a um aumento de cerca de $275,8 \%(P<0,0001)$.

No teste oral de tolerância à glicose, a área sob a curva foi maior no grupo hiperlipídico comparado ao grupo controle ( $+297,6 \%, P<0,001$ - GC:14677u.a.; GH:58361u.a.) (Figura 4), confirmando a diabetes em todas as ratas.

\section{Discussão}

O regime dietético empregado no presente estudo exemplifica a situação nutricional vivenciada pela

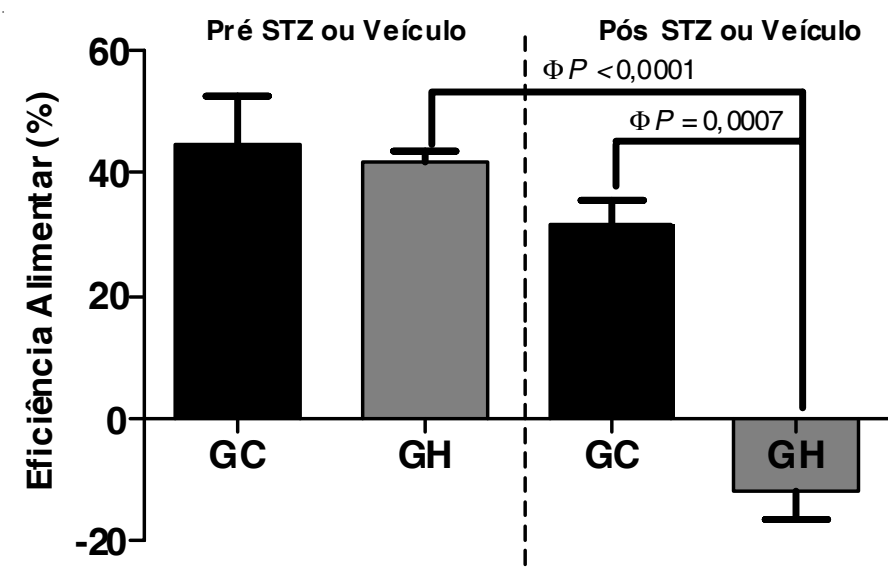

Figura 1: Eficiência alimentar dos diferentes grupos experimentais, nos períodos pré injeção de estreptozotocina (STZ) ou veículo e pós injeção de STZ e veículo. Grupos: GC, grupo controle e GH, grupo hiperlipídico. O Símbolo ( $\phi$ ) representa diferença estatística.

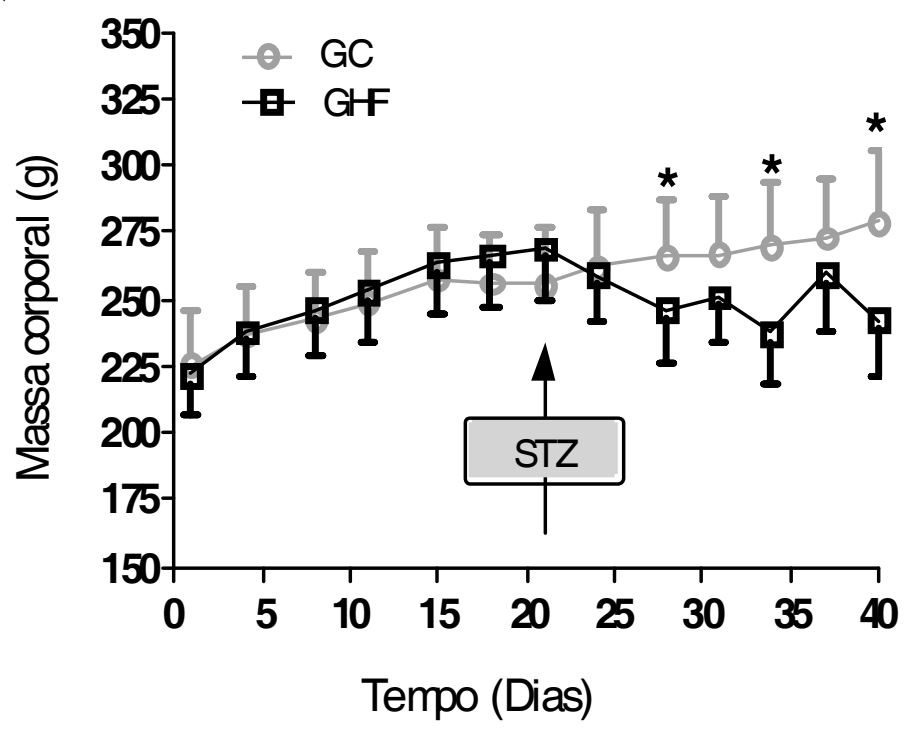

Figura 2: Evolução da massa corporal ao logo da primeira fase do experimento (indução). As dietas foram administradas durante 3 semanas e ao final da última semana foi administrada intraperitonealmente estreptozotocina (STZ) ao grupo hiperlipídico e veículo ao grupo controle. Grupos: GC, grupo controle e GHF, grupo hiperlipídico. O símbolo "*" representa diferença estatística, $P<0,001$.

população mundial, uma vez que há oferta abundante de alimentos hiperlipídicos. ${ }^{11}$ A utilização de ácidos graxos saturados como fonte predominante de lipídios vem sendo associada não somente à obesidade, mas também ao desenvolvimento de resistência à insulina, intolerância a glicose e desordens no metabolismo de lipoproteínas ${ }^{12}$, sendo este o fundamento para sua utilização em pesquisas relacionadas ao diabetes.

Nesta linha de pensamento, tentou-se reproduzir um modelo experimental, não genético, que mime- 

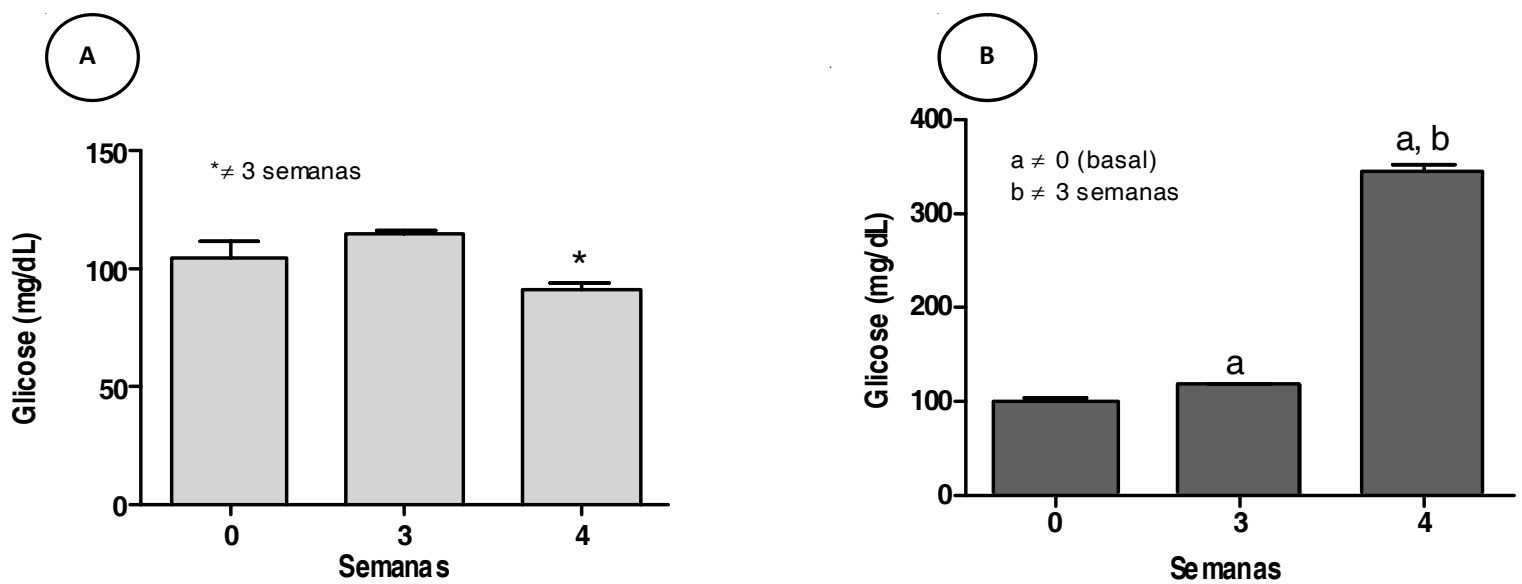

Figura 3: Contrações médias de glicose nos grupos experimentais. (A) Grupo Controle, concentrações médias basais de glicose (0 zero), após três semanas de administração de dieta controle e após injeção i.p. de veículo (4 semanas). (B) Grupo Hiperlipídico, concentrações médias basais de glicose, após três semanas de administração de dieta hiperlipídica e após injeção i.p. de estreptozotocina. O símbolo (*) e as letras (a; b) representam diferença estatística, $P<0,01$.
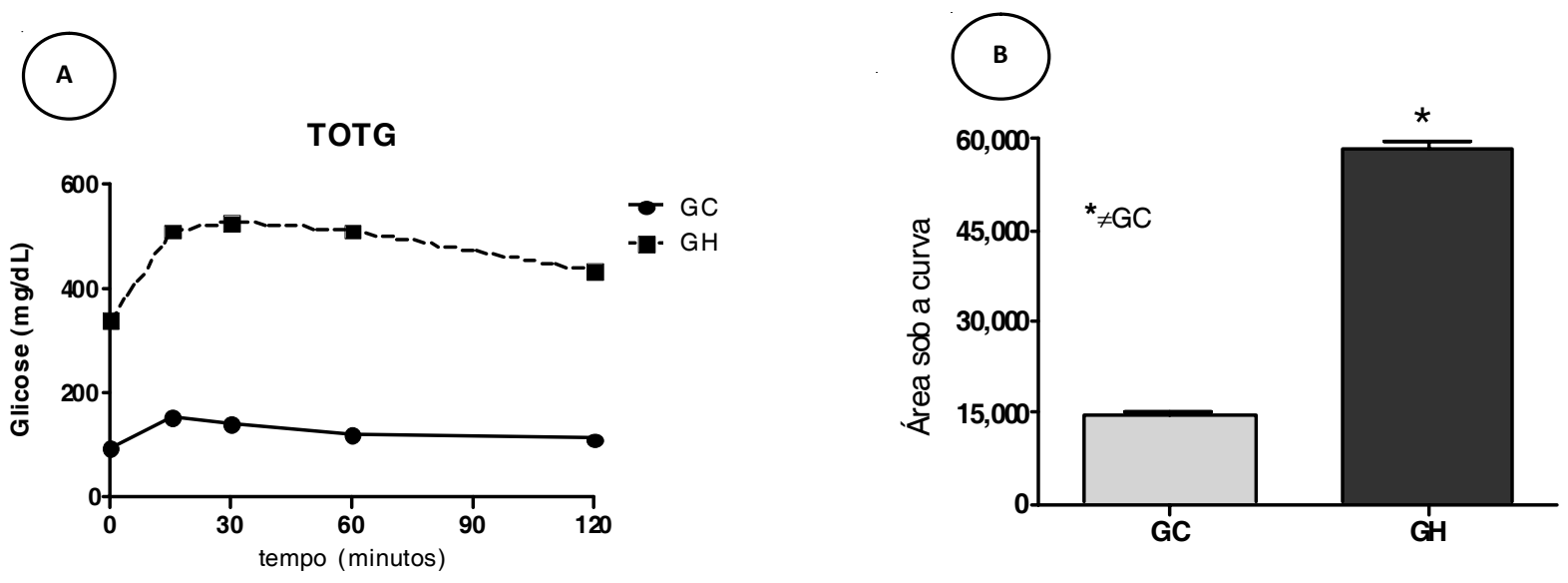

Figura 4: Teste oral de tolerância glicose após uma semana de administração de estreptozotocina ou veículo. As curvas glicêmicas de resposta à sobrecarga de glicose são mostradas em (A), grupo controle e grupo hiperlipídico, respectivamente e as áreas sob as curvas são mostradas em (B). O Símbolo $\left(^{*}\right)$ representa diferença estatística, $P<0,0001$.

tizasse o curso natural e metabólico da diabetes tipo 2 em humanos (combinação de resistência à insulina e deficiência de insulina). Para tal, administrou-se dieta hiperlipídica, rica em ácidos graxos saturados e pobre em carboidratos, durante três semanas, com a finalidade de causar a resistência à insulina, e em seguida foi injetada uma dose baixa e única de STZ, levando à lesão das células $\beta$ (deficiência de insulina), seguindo por mais uma semana de dieta hiperlipídica.

Analisando os resultados ao longo do período de experimento, referentes à ingestão alimentar, percebemos que durante as três semanas iniciais do estudo os animais que foram alimentados com a dieta hiperlipídica apresentaram menor ingestão alimentar quando comparados aos animais do grupo controle. Hariri et al. ${ }^{13}$ em sua pesquisa encontraram resulta- dos semelhantes em relação ao consumo de ração, onde ofertaram dietas hiperlipídicas (67\% da energia total) com diferentes fontes de lipídio (banha e óleo de canola) a ratos Sprague-Dawley por um período de 26 dias, a fim de analisar o efeito da dieta como facilitador ao ganho de peso excessivo. Além disso, a ingestão energética foi superior no grupo alimentado com a ração que continha a banha de porco, semelhante aos nossos animais, e da mesma forma ambos os grupos apresentaram redução na ingestão alimentar no momento de transição da dieta padrão (utilizada no período de ambientação) para a oferta das dietas hiperlipídicas, porém sem diferença significativa. Vale ressaltar que lipídios estimulam a liberação de colecistoquinina (hormônio da saciedade) $)^{14}$ e a redução de consumo dos animais que consumiram dieta hiper- 
lipídica pode ser devido a este fato. No mesmo estudo $^{13}$, também foi observado, que ao comparar os grupos hiperlipídicos (óleo de canola ou manteiga) com o grupo controle, este apresentou maior ingestão alimentar. Em ambos os estudos, a semelhança da redução na ingestão dietética pode estar relacionada com uma adaptação dos animais em conseqüência a alterações na qualidade da alimentação, inicialmente composta pela dieta padrão, modificando-se para dieta hiperlipídica, a qual segue fornecendo maior densidade energética com possíveis alterações sensoriais, além da suposta saciedade já mencionada.

Podemos observar que no período seguido à administração da STZ e veículo, tanto o grupo hiperlipídico quanto o grupo controle, respectivamente, apresentaram redução da ingestão alimentar e igualaramse na quantidade de ração consumida. Contrapondose aos resultados do presente experimento, Yokoyama et al. ${ }^{15}$, utilizando ratos machos Sprague- Dawley, observaram que os animais diabéticos induzidos por STZ apresentaram ingestão alimentar estatisticamente superior, porém com semelhança nos dados encontrados referentes ao ganho de peso e eficácia alimentar, onde estes apresentaram valores significativamente menores em relação aos animais não diabéticos. Neste estudo, a eficiência alimentar durante o período inicial foi igual em ambos os grupos, tornando-se negativa após administração da STZ no grupo hiperlipídico.

O balanço energético positivo contribui para o ganho de peso corporal, mesmo a ingestão alimentar sendo relativamente pequena em termos quantitativos, porém elevada em termos de calorias. Um padrão dietético constituído em sua maioria por alimentos com elevada densidade energética, é um dos fatores mais relevantes para o ganho de peso excessivo. ${ }^{16,17}$ Os animais do grupo hiperlipídico não apresentaram diferença significativa na massa corporal em relação aos animais do grupo controle no período em que estavam sendo ofertadas apenas as dietas experimentais. Com o objetivo de reproduzir um modelo animal, em fêmeas Sprague-Dawley, de obesidade a partir de estímulos dietéticos, Hariri et al. ${ }^{13}$ observaram que o grupo alimentado com a banha ao final deste período apresentou maior peso corporal, porém sem valores significativos; semelhante aos nossos resultados.

Ainda em relação ao ganho de peso dos animais, após a administração da STZ, foi observada uma redução na massa corporal dos mesmos, sem ganho ao longo do experimento. A perda de peso encontrada nos animais após a injeção de STZ já era esperada, pois esta, em ratos, leva ao desenvolvimento de uma síndrome clínica caracterizada por hiperglicemia e poliúria, que também ocorreu em nossos animais, além perda de peso. ${ }^{18}$ Estes resultados corroboram com outros estudos onde também foi encontrada perda de peso após a administração de diferentes doses de STZ em animais. ${ }^{2,19,20,21}$ Sabe-se que a perda de peso é uma característica do estado de hiperglicemia aguda em humanos, entre outros sintomas clássicos. ${ }^{22} \mathrm{O}$ paciente diabético exibe um quadro em que há deficiência na produção de insulina, e como consequiência a utilização da glicose pelos músculos e tecido adiposo encontra-se prejudicada. A mesma é liberada pelo pâncreas em resposta aos níveis aumentados de glicemia após as refeições com objetivo de armazenar energia. ${ }^{23}$ Porém, com o indivíduo apresentando uma reduzida razão entre insulina e glucagon, haverá um estímulo para a gliconeogênese hepática e cetogênese, com maior degradação de lipídeos e proteínas, que serão usados como fontes alternativas de energia, já que a glicose não consegue ser plenamente utilizada. Tal processo é semelhante às consequiências do estado de jejum, porém com a diferença dos níveis elevados de glicemia. A utilização destas fontes alternativas gera como consequiência a perda de peso, pela redução da massa de tecido adiposo utilizado como principal fonte energética. ${ }^{24}$

A deficiência de insulina está geralmente associada com atrofia muscular esquelética. Estudo realizado por Pepato et al..$^{25}$, mostrou que, após a indução com STZ, ocorrem variações temporais na atividade das diferentes vias proteolíticas no músculo, sendo observado aumento na proteólise muscular na fase aguda e uma redução em períodos mais prolongados de deficiência de insulina. Desta forma, a redução das reservas lipídicas, associada a um aumento no catabolismo muscular em consequiência da maior degradação das proteínas estruturais, pode ser uma das explicações da perda de massa corporal dos animais diabéticos do presente estudo. ${ }^{26}$

Resultados semelhantes foram encontrados no experimento realizado por Yang et al. ${ }^{27}$, em que utilizaram machos Sprague- Dawley, e da mesma forma induziram a diabetes utilizando dieta hiperlipídica e STZ em baixa dose (duas vezes na semana e por duas semanas). O grupo de animais diabéticos induzidos pela dieta hiperlipídica e STZ apresentou menor peso corporal do que o grupo controle, e ambos apresentaram peso final menor em relação ao grupo diabético induzido apenas pela dieta hiperlipídica, porém sem valores significativos entre os grupos ao final de 12 semanas. 
Após o período de administração da dieta hiperlipídica, observou-se um aumento significativo da glicemia de jejum, em comparação aos animais que receberam a dieta controle. A literatura ainda apresenta resultados divergentes. McDonald et al. ${ }^{28}$, utilizando fêmeas wistar, não observaram diferença na glicemia de jejum entre os grupos por eles analisados. Em seu estudo, foi ofertada uma dieta hipercalórica rica em lipídio ( $41 \%$ da energia total) por um longo período, identificando-se apenas um aumento gradual ao comparar diferentes momentos do experimento (7, 26, 39 semanas). Entretanto, tais autores também ressaltam que mesmo não havendo diferença significativa do peso corporal entre os distintos grupos, houve modificações nos compartimentos de gordura, sendo este um fator de risco para desenvolvimento de distúrbios metabólicos.

Fujimoto et al..$^{29}$, utilizando machos SpragueDawley, encontraram valores de glicemia de jejum e de insulina superiores nos animais que foram submetidos a 77 dias de indução da resistência à insulina através da dieta hiperlipídica. Estes autores também determinaram a área sob a curva da glicose, onde foi maior nos animais alimentados com a dieta rica em lipídio, em relação aos animais que receberam dieta padrão, apresentando características de intolerância a glicose, analisada através do TOTG.

$\mathrm{O}$ aumento da glicemia no nosso estudo pode ser explicado pelo fato de que os ácidos graxos, combustível essencial da célula beta no estado normal, também se tornam tóxicos à célula beta quando presentes cronicamente em níveis elevados. A exposição prolongada das células $\beta$ aos ácidos graxos aumenta a secreção basal de insulina inibindo, porém, a secreção de insulina estimulada pela glicose. A lipotoxicidade é mediada provavelmente pelo acúmulo de um sinal citosólico derivado da via de esterificação do ácido graxo. Os ácidos graxos inibem a expressão do gene da insulina na presença de níveis elevados de glicose, em parte devido à regulação negativa do fator de transcrição pancreático duodenal homeobox-1. Ácidos graxos em excesso também levam a morte da célula $\beta$ por apoptose tanto in vivo como in vitro. ${ }^{30}$

No período seguinte a associação da dieta hiperlipídica somada à administração da STZ, os animais apresentaram um aumento significativo da glicemia, evidenciando a ação da STZ em relação ao metabolismo da insulina. Segundo Davidson et al. ${ }^{31}$, que de forma semelhante, utilizando ratos Sprague Dawley, trabalharam com modelo de DM2 induzido a partir de dieta high-fat juntamente com ação da STZ, encontraram resultados similares em relação à glicemia de jejum, sendo esta superior em relação aos animais não induzidos; e, indo de encontro aos nosso resultados também apresentaram um menor ganho de peso corporal dos animais classificados como diabéticos.

\section{Conclusão}

O presente estudo demonstrou que a combinação de dieta hiperlipídica e baixa dose de STZ por via intraperitoneal foi eficaz em gerar um modelo animal que reproduz a história natural e as características metabólicas comuns da diabetes tipo 2 em humanos. Esse modelo é de baixo custo, fácil e mais adequado para o estudo da patofisiologia da diabetes tipo 2 .

\begin{abstract}
The objective of the present experimental study is to develop an animal model of type 2 diabetes which mimics the natural and metabolic course of this disease in humans. Therefore, a high-fat diet (with approximately $60 \%$ of the calories from lipidis) was offered for 3 weeks, establishing an insulin resistant picture. After, the female rats were submitted to a single dose of streptozotocin (STZ) (35mg/kg of body mass) using citrate buffer $(\mathrm{pH}: 4,4)$. After a week of the injection of STZ, the female rats were submitted to the glucose tolerance oral test, by oral administration of glucose ( $2 \mathrm{~g}$ glucose/ $\mathrm{kg}$ of body mass). The data was compared between groups using the test $t$. The significance in all tests was on level of $p<0,05$. The control group kept the final average glucose concentration equals to the initial. After the ingestion of the high-fat diet an increase of $16,5 \%$ of the fasting glucose happened compared to the initial moment of the experiment $(P<0,0001)$ while the animals that received the control diet presented an increase of approximately $8,7 \%$. One week after the intraperitoneal administration of STZ, the high-fat feed group showed an increase of about $194,2 \%$ of the average concentration of glucose, representing an increase of about $275,8 \%(P<0,0001)$ when compared to the control group. The present study shows that the combination of a high-fat diet and low dose of streptozotocin works as an alternative animal model for type 2 diabetes simulating the disease in humans.
\end{abstract}

Key-words: Diabetes Mellitus, Experimental. Streptozotocin. Rats, Wistar. Diet, High-Fat. 


\section{Referências Bibliográficas}

1. McLellan KCP, Barbalho SM, Cattalini M, Lerario AC. Diabetes mellitus do tipo 2, síndrome metabólica e modificação no estilo de vida. Rev Nutr. 2007; 20: 515-24.

2. Srinivasan K, Viswanad B, Asrat L, Kaul CL, Ramarao P. Combination of high-fat diet and low-dose of streptozotocintreated rat: A model for type 2 diabetes and pharmacological screening. Pharmacol Res. 2005; 52: 313-20.

3. Avramoglu KR, Basciano H, Adeli K. Lipid and a lipoprotein dysregulation in insulin resistant states. Clin Chim Acta. 2006; $368: 1-19$.

4. Saltiel AR. New perspectives into the molecular pathogenesis and treatment of type 2 Diabetes. Cell. 2001; 23: 517-29.

5. Petersen FK, Shulman IG. Pathogenesis of skeletal muscle insulin resistance in type 2 diabetes mellitus. Am $\mathrm{J}$ Cardiol. 2002; 90: 11G-18G.

6. Nolan CJ, Damn P, Prentki M. Type 2 diabetes across generations: from pathophysiology to prevention and management. Lancet. 2011; 378(9786): 169-81.

7. Kirsten VR, Sesterhein P, Saitovitch D. Modelos experimentais para o estudo do diabetes tipo 1. Medicina (Ribeirão Preto) 2010; 43: 3-10.

8. Mu J, Woods J, Zhou YP, Roy RS, Li Z, Zycband E, et al. Chronic inhibition of dipeptidyl peptidase- 4 with a sitagliptin analog preserves pancreatic $\beta$-cell mass and function in a rodent model of type 2 diabetes. Diabetes. 2006; 55: 16951704.

9. Wang Y, Wang P, Qin L, Davaasambuu G, Kaneko T, Xu J, et al. The development of diabetes mellitus in wistar rats kept on a high-fat/low-carbohydrate diet for long periods. Endocrine. 2003; 22: 85-92.

10. Yu Q, Larson DF, Slayback D, Lundeen TF, Baxter JH, Watson RR. Characterization of high-salt and high-fat diets on cardiac and vascular function in mice. Cardiovasc Toxicol. 2004; 4: 37-46.

11. Manco M, Calvani M, Mingrone G. Effects of dietary fatty acids on insulin sensitivity and secretion. Diabetes Obes Metab 2004; 6: 402-13.

12. Lima FEL, Menezes TN, Tavares MP, Szarfarc SC, Fisberg RM. Ácidos graxos e doenças cardiovasculares: uma revisão. Rev Nutr. 2000; 13: 73-80.

13. Hariri N, Gougeon R, Thibault L. A highly saturated fat-rich diet is more obesogenic than diets with lower saturated fat content. Nutr Res. 2010; 30: 632-43.

14. Rosado EL, Monteiro JBR. Obesidade e a substituição de macronutrientes da dieta. Rev Nutr. 2001; 14: 145-52.

15. Yokoyama M, Tanigawa K, Murata T, Kobayashi Y, Tada E, Suzuki I, et al. Dietary polyunsaturated fatty acids slow the progression of diabetic nephropathy in streptozotocin-induced diabetic rats. Nutr Res. 2010; 30: 217-25.

16. Hill JO, Wyatt HR, Reed GW, Peters JC. Obesity and the environment: where do we go from here? Science. 2003; 299(5608):853-5.
17. Jéquier E. Pathways to obesity. Int J Obes Relat Metab Disord 2002; 26 (Suppl 2): S12-S17.

18. Schaan BD, Dall'Ago P, Maeda CY, Ferlin E, Fernandes TG, Schmid $\mathrm{H}$, et al. Relationship between cardiovascular dysfunction and hyperglycemia in streptozotocin-induced diabetes in rats. Braz J Med Biol Res. 2004; 37: 1895-902.

19. Motyl K, McCabe LR. Streptozotocin, Type I diabetes severity and bone. Biol Proced Online. 2009; 6: 296-315.

20. Tian H, Wei L, Xu Z, Zhao R, Jin D, Gao J. Correlations between blood glucose level and diabetes signs in streptozotocin-induced diabetic mice. Global J Pharmacol. 2010; 4: 111-16.

21. Silva M, Lima WG, Silva ME, Pedrosa ML. Efeito da estreptozotocina sobre os perfis glicêmico e lipídico e o estresse oxidativo em hamsters. Arq Bras Endocrinol Metab. 2011; 55: 46-53.

22. American Diabetes Association. Report of the expert committee on the diagnosis and classification of diabetes mellitus. Diabetes Care. 2003; 26(Suppl 1): S5-20.

23. Shils ME, Shike M, Ross AC, Caballero B, Cousins RJ. Nutrição Moderna. Na Saúde e na Doença. $10^{\circ}$ ed. Barueri, São Paulo: Manole, 2009.

24. Berg JM, Tymoczka JL, Stryer L. Bioquímica. $6^{\circ}$ ed. Rio de Janeiro: Guanabara Koogan, 2008.

25. Pepato MT, Migliorini RH, Goldberg AL, Kettelhut IC. Role of different proteolytic pathways in degradation of muscle protein from streptozotocin-diabetic rats. Am J Physiol Endocrinol Metab. 1996; 271: E340- 7.

26. Ramachandran S, Rajasekaran A, Kumar KTM. Antidiabetic, antihyperlipidemic and antioxidant potential of methanol extract of Tectona grandis flowers in streptozotocin induced diabetic rats. Asian Pac J Trop Med. 2011;4:624-31.

27. Yang J, Campitelli J, Hu G, Lin Y, Luo J, Xue C. Increase in DPPIV in the intestine, liver and kidney of the rat treated with high fat diet and streptozotocin. Life Sci. 2007; 81: 272-9.

28. Mcdonald SD, Pesarchuk E, Don-Wauchope A, Zimaity HE, Holloway AC. Adverse metabolic effects of a hypercaloric, high-fat diet in rodents precede observable changes in body weight. Nutr Res. 2011; 31: 707-14.

29. Fujimoto S, Mochizuki K, Shimada M, Hori T, Murayama Y, Ohashi N, Goda T. Insulin resistance induced by a high-fat diet is associated with the induction of genes related to leukocyte activation in rat peripheral leukocytes. Life Sci. 2010; 87: 679-85.

30. Donath MY, Shoelson ES. Type 2 diabetes as an inflammatory disease. Nat Rev Immunol. 2011; 11: 98-107.

31. Davidson EP, Coppey LJ, Holmes A, Yorek MA. Effect of inhibition of angiotensin converting enzyme and/or neutral endopeptidase on vascular and neural complications in high fat fed/low dose streptozotocin-diabetic rats. Eur J Pharmacol. 2012; 677: 180-7. 